Published in final edited form as:

Science. 2008 March 21; 319(5870): 1668-72. doi:10.1126/science.1154584.

\title{
TDP-43 Mutations in Familial and Sporadic Amyotrophic Lateral Sclerosis
}

\author{
Jemeen Sreedharan ${ }^{\# 1}$, lan P. Blair ${ }^{\# 3,4}$, Vineeta B. Tripathi ${ }^{\# 1}$, Xun $\mathrm{Hu}^{1}$, Caroline Vance ${ }^{1}$, \\ Boris Rogelj $^{1}$, Steven Ackerley ${ }^{1,2}$, Jennifer C. Durnall ${ }^{3}$, Kelly L. Williams ${ }^{3}$, Emanuele \\ Buratti $^{5}$, Francisco Baralle ${ }^{5}$, Jacqueline de Belleroche ${ }^{6}$, J. Douglas Mitchell ${ }^{7}$, P. Nigel \\ Leigh $^{1}$, Ammar Al-Chalabi ${ }^{1}$, Christopher C. Miller ${ }^{1,2}$, Garth Nicholson ${ }^{\# 3,4,8}$, Christopher E. \\ Shaw $^{\# 1, \dagger}$ \\ ${ }^{1}$ Department of Clinical Neuroscience, King's College London, Medical Research Council (MRC) \\ Centre for Neurodegeneration Research, and Institute of Psychiatry, London SE5 8AF, UK \\ 2Department of Neuroscience, King's College London, MRC Centre for Neurodegeneration \\ Research, and Institute of Psychiatry, London SE5 8AF, UK \\ ${ }^{3}$ Northcott Neuroscience Laboratory, ANZAC Research Institute, Concord, NSW, 2137, Australia \\ ${ }^{4}$ Faculty of Medicine, University of Sydney, NSW, 2139, Australia \\ ${ }^{5}$ International Centre for Genetic Engineering and Biotechnology, Padriciano 99, 34012 Trieste, \\ Italy
}

${ }^{6}$ Division of Neurosciences and Mental Health, Faculty of Medicine, Imperial College London, and Charing Cross Hospital, London W6 8RF, UK

${ }^{7}$ Department of Neurology, Royal Preston Hospital, Preston PR2 9HT, UK

${ }^{8}$ Molecular Medicine Laboratory, Concord Repatriation General Hospital, Concord, NSW, 2139, Australia

\# These authors contributed equally to this work.

\begin{abstract}
Amyotrophic lateral sclerosis (ALS) is a fatal motor neuron disorder characterised pathologically by ubiquitinated TAR DNA binding protein (TDP-43) inclusions. The function of TDP-43 in the nervous system is uncertain and a mechanistic role in neurodegeneration remains speculative. We identified neighbouring mutations in a highly conserved region of TARDBP in sporadic and familial ALS cases. TARDBP ${ }_{M 337 V}$ segregated with disease within one kindred and a genomewide scan confirmed that linkage was restricted to chromosome $1 \mathrm{p} 36$, which contains the TARDBP locus. Mutant forms of TDP-43 fragmented more readily than wild-type in vitro and caused neural apoptosis and developmental delay in the chick embryo in vivo. Our evidence suggests a pathophysiological link between TDP-43 and ALS.
\end{abstract}

\footnotetext{
${ }^{\dagger}$ To whom correspondence should be addressed.chris.shaw@iop.kcl.ac.uk.
} 
ALS (also known as Lou Gehrig's disease) is a relentlessly progressive and ultimately fatal adult-onset disorder characterised pathologically by the degeneration of motor neurons in the brain and spinal cord (1). ALS is familial in 5-10\% of cases (FALS) with an autosomal dominant pattern of inheritance. Mutations in $\mathrm{CuZn}$ superoxide dismutase (SOD1) are known to cause ALS, and are detected in $~ 20 \%$ of FALS and $3 \%$ of sporadic ALS (SALS) cases $(2,3)$. Mice transgenic for mutant human SOD1 develop selective motor neuron degeneration due to a toxic gain of function (4). Pure FALS kindreds have been linked to chromosome 18q (5), 16q (6) and 20p (7). Other dominant kindreds that have a phenotypic spectrum ranging from pure ALS to pure frontotemporal lobar dementia (FTLD) and individuals who have features of both disorders have been linked to chromosome $9 p$ and $9 q$ (8-10), but no pathogenic mutations have been identified in these kindreds.

The presence of ubiquitinated inclusions (UBIs) in the perikaryon and proximal axon of surviving spinal motor neurons is the pathological hallmark of ALS and indicates a failure of the proteasome to recycle damaged proteins (11). UBIs are also prominent in cortical neurons within the frontal and temporal lobes in patients with tau-negative frontotemporal lobar dementia (FTLD-U). Many individuals who present with a pure ALS phenotype will have pathological features of FTLD-U and vice versa, again providing a circumstantial link between FTLD and ALS (12). The TAR DNA binding protein (TDP-43) is the major protein in UBIs in FTLD-U and ALS $(13,14)$. A phosphorylated 25-kD C-terminal fragment of TDP-43 and high-molecular weight ubiquitinated aggregates are enriched in detergent resistant fractions of FTLD-U and ALS brains. Intriguingly, those neurons with cytoplasmic inclusions have a striking loss of nuclear TDP-43 raising the possibility that cleavage of full length TDP-43 and cytoplasmic sequestration might play a mechanistic role in neurodegeneration. TDP-43 inclusions are seen in glia and its deposition has been observed in many different neurodegenerative disorders (15-18) and claims of a pathophysiological role for TDP-43 aggregation in ALS have been challenged (19).

To investigate the role of TDP-43 in ALS pathogenesis we screened 154 index FALS cases for mutations in TARDBP, the gene encoding TDP-43. SOD1, VAPB, ANG, Dynactin and $C H M P 2 B$ mutations had been excluded from these cases. We identified a missense mutation in exon 6 of TARDBP in the index case from kindred ALS85 (Fig. 1A), a Caucasian family of English descent. The mutation is predicted to result in substitution of valine for methionine (M337V) and resides in a strongly phylogenetically conserved region of TDP-43 (Fig. 1C). The mutation segregated with disease, being present in four other affected individuals in three branches and two generations of the extended kindred, and absent from nine unaffected siblings.

Family ALS85 demonstrated autosomal dominant inheritance with male to male transmission apparent. Four of the affected individuals had definite ALS by the El-Escorial criteria (20) and another recently symptomatic individual had probable ALS. Three had limb-onset ALS and two had bulbar-onset ALS. The mean age of symptom onset was 47 years (range 44 - 52). Mean disease duration was 5.5 years (range 4 - 7) from symptom onset to death. The obligate carrier (who died aged 54 from severe coronary atheroma) was reported by family members to have had gait disturbance and declining upper limb strength 
consistent with ALS. There was no history of dementia or any atypical features in the kindred.

We next conducted a genome-wide scan in this kindred. DNA was available from 23 members of the kindred including 5 affected individuals (4 males and one female). By analysis of single-nucleotide polymorphism (SNP) genotypes we identified linkage to chromosome 1p36, which contains the TARDBP locus, with a parametric multipoint LOD score of 2.04 (Fig. 2A). Fine mapping across the region with microsatellite markers confirmed linkage with a maximal two point LOD score of 2.73 at D1S450 (table S1) and a highly significant multipoint LOD score of 2.93 (Fig. 2B). These LOD scores approach the simulated maximum power to detect linkage in this kindred (simulated maximal LOD score of 2.98). Only one other locus on chromosome 15 generated a LOD score > 1 (fig. S1) but fine mapping with microsatellite markers confidently excluded linkage to this region (table S1). Analysis of the microsatellite markers spanning the chromosome $1 \mathrm{p} 36$ region revealed a clear haplotype that segregated with the mutation and disease (Fig. 2C). Recombinant events in two unaffected individuals (II:4 and II:8) defined the telomeric limit as D1S2795.

Recombination in one affected individual (III:8) defined the centromeric limit as D1S228. This linked region spanning $16.5 \mathrm{cM}(\sim 8.2 \mathrm{Mb})$ contains 97 genes in total including TARDBP and the $1009 \mathrm{~A}>\mathrm{G}$ mutation.

We next sought to determine the frequency of TDP-43 mutations by sequencing all six exons in a cohort of 200 British SALS cases. We identified a missense mutation in exon 6 (991 $\mathrm{C}>\mathrm{A}$ ) which is 18 base pairs upstream of the familial mutation and predicted to substitute lysine for glutamine (Q331K) (Fig. 1B). This mutation was found in a man who developed limb-onset ALS aged 72 with disease duration of three years. No mutations were detected in a screen of all six exons of TARDBP from 500 British Caucasian controls, emphasising the high degree of evolutionary conservation. Because Q331K and M337V are unlikely to be benign polymorphisms we proceeded to study the functional effect of these two mutations.

Prior to manuscript submission we screened all 6 exons of TARDBP in two further cohorts: 172 Australian Caucasian SALS cases and 172 controls, as well as 200 British Caucasian controls. A further mutation was detected in exon 6 in an Australian SALS case (881 G>C) predicted to substitute alanine for glycine (G294A) (Fig 1B). The patient was a man who developed limb onset ALS aged 65 with disease duration of 5 years and no atypical features. One non-synonymous change in a single control was identified in exon $3(269 \mathrm{C}>\mathrm{T})$ predicted to substitute alanine for valine (A90V) (Fig. S2). This individual was a healthy 53 year old Caucasian male. Lastly, we sequenced exon 6 of TARDBP in an additional 390 British and Australian Caucasian controls and found no mutations. Mutations were detected in exon 6 in 3/526 ALS cases and 0/1,262 controls ( $P=0.025$ by Fischer Exact Test).

In order to assess the functional significance of these mutations we expressed tagged TDP- $43_{\mathrm{WT}}, \mathrm{TDP}-43_{\mathrm{Q} 331 \mathrm{~K}}$ and TDP-43 $3337 \mathrm{~V}$ in chinese hamster ovary $(\mathrm{CHO})$ cells. Immunofluorescent staining of cells 48 hours after transfection demonstrated abundant expression of transfected TDP-43. No obvious differences in subcellular distribution or aggregation were observed between wild-type and mutant proteins. Using the lactate dehydrogenase (LDH) assay there was no significant increase in cell death in CHO cells 
transfected with either TDP-43 mutant when compared to wild-type. Probing immunoblots of the cytoplasmic fraction for the $\mathrm{N}$-terminal myc tag revealed numerous fragments varying in molecular weight from $\sim 14-45 \mathrm{kD}$ (Fig. 3A). Quantitative analysis of the most prominent bands demonstrated a non-significant increase in the $14 \mathrm{kD}$ band in the mutants and a significant increase in the $18 \mathrm{kD}$ band when compared to wild-type TDP-43 (Fig. 3B, WT v Q331K, $P<0.01$, WT v M337V, $P<0.05)$.

We next investigated the effects of TDP-43 mutants in chick embryos. This model allows DNA to be efficiently delivered directly into the neural tube by electroporation, permitting in vivo analysis of neural tissue expressing transfected genes (21). We expressed tagged TDP-43 ${ }_{\mathrm{WT}}$, TDP- $43_{\mathrm{Q} 331 \mathrm{k}}$ and TDP-43 $\mathrm{M} 337 \mathrm{~V}$ in the spinal cords of Hamburger Hamilton stage 14 chick embryos (Fig. 4, D-F). SOD1 $1_{\mathrm{WT}}$ and the ALS-linked SOD1 $1_{\mathrm{G} 93 \mathrm{~A}}$ mutant were used as controls. Embryos expressing TDP- $43_{\mathrm{Q} 331 \mathrm{~K}}$ and TDP-43 ${ }_{\mathrm{M} 337 \mathrm{~V}}$ showed a dramatic reduction in maturation compared to TDP- $43_{\mathrm{WT}}$ with a failure to develop normal limb and tail buds (Fig. 4, A-C). Chick embryo development proceeded normally over 48 hours with TDP- $43_{\mathrm{WT}}, \mathrm{SOD} 1_{\mathrm{WT}}$ and $\mathrm{SOD} 1_{\mathrm{G} 93 \mathrm{~A}}$ but at 24 hours only $5-15 \%$ of those embryos expressing mutant TDP-43 had reached the normal stage of maturation (Fig. $4 \mathrm{~J}, P<10^{-14}$ ). Terminal deoxynucleotidyl transferase-mediated dUTP-biotin nick end labelling (TUNEL) staining demonstrated a significant increase in the number of apoptotic nuclei in embryos expressing either mutant TDP-43 when compared to wild-type (Fig. 4, G-I and K, $P<$ 0.001). These results suggest a toxic gain of function or dominant negative effect of mutant TDP-43.

TDP-43 is a ubiquitously expressed nuclear protein capable of binding DNA and RNA, which regulates transcription and splicing but may also be involved in microRNA biogenesis, apoptosis and cell division (22). The C-terminal domain binds heterogeneous ribonuclear proteins and inhibits splicing of the cystic fibrosis transmembrane conductance regulator mRNA (23). The accumulation of hyperphosphorylated TDP-43 fragments in the perikaryon of neurons in FTLD-U and ALS is accompanied by a striking loss of TDP-43 from the nucleus $(13,14)$. The sequestration of TDP-43 would be predicted to disrupt the regulation of transcription and splicing, which may account for the 1.5 fold increase in TDP-43 mRNA seen in FTLD-U brains (24). The identification of TDP-43 mutations that result in increased fragmentation and toxicity to neural cells strongly supports a pathophysiological role for TDP-43 misaccumulation in ALS. Robust linkage to the TARDBP locus in kindred ALS85 adds crucial evidence that the M337V mutation is pathogenic. The G294A, Q331K and M337V mutations are all localized to a highly conserved region of the C-terminus of TDP-43 known to be involved in protein-protein interactions (23). The Q33 1K mutation in particular creates a new protein kinase A site, which may result in abnormal phosphorylation. The G294 A mutation interrupts a glycine run motif characteristic of all hnRNPs and may interfere with RNA binding and gene suppression (25).

Three other studies have failed to identify TARDBP mutations in FTLD and ALS (26-28). Our findings suggest that although mutations are rare they may be pathogenically linked to ALS. Parallels exist with other neurodegenerative disorders where the identification of rare familial mutations in the amyloid precursor protein in Alzheimer disease and a-synuclein in 
Parkinson disease has dramatically advanced studies into the pathogenesis of a predominantly sporadic disease. Elucidating the biochemical processes responsible for the increased fragmentation of TDP-43 and its toxicity to neural tissues may provide important insights into disease mechanisms that underlie ALS.

\section{Supplementary Material}

Refer to Web version on PubMed Central for supplementary material.

\section{References and Notes}

1. Shaw CE, Al-Chalabi A, Leigh N. Curr Neurol Neurosci Rep. 2001; 1:69. [PubMed: 11898502]

2. Rosen DR, et al. Nature. 1993; 362:59. [PubMed: 8446170]

3. Shaw CE, et al. Neurology. 1997; 49:1612. [PubMed: 9409355]

4. Gurney ME, et al. Science. 1994; 264:1772. [PubMed: 8209258]

5. Hadano S, et al. Nat Genet. 2001; 29:166. [PubMed: 11586298]

6. Ruddy DM, et al. Am J Hum Genet. 2003; 73:390. [PubMed: 12840784]

7. Sapp PC, et al. Am J Hum Genet. 2003; 73:397. [PubMed: 12858291]

8. Hosler BA, et al. JAMA. 2000; 284:1664. [PubMed: 11015796]

9. Vance C, et al. Brain. 2006; 129:868. [PubMed: 16495328]

10. Morita M, et al. Neurology. 2006; 66:839. [PubMed: 16421333]

11. Leigh PN, et al. Brain. 1991; 114(Pt 2):775. [PubMed: 1646064]

12. Lipton AM, White CL III, Bigio EH. Acta Neuropathol. 2004; 108:379. [PubMed: 15351890]

13. Neumann M, et al. Science. 2006; 314:130. [PubMed: 17023659]

14. Arai T, et al. Biochem Biophys Res Commun. 2006; 351:602. [PubMed: 17084815]

15. mador-Ortiz C, et al. Ann Neurol. 2007; 61:435. [PubMed: 17469117]

16. Hasegawa M, et al. Brain. 2007; 130:1386. [PubMed: 17439983]

17. Nakashima-Yasuda H, et al. Acta Neuropathol. 2007; 114:221. [PubMed: 17653732]

18. Freeman SH, Spires-Jones T, Hyman BT, Growdon JH, Frosch MP. J Neuropathol Exp Neurol. 2007

19. Rothstein JD. Ann Neurol. 2007; 61:382. [PubMed: 17469124]

20. Brooks BR, Miller RG, Swash M, Munsat TL. Amyotroph Lateral Scler Other Motor Neuron Disord. 2000; 1:293. [PubMed: 11464847]

21. Ghadge GD, et al. Neurobiol Dis. 2006; 21:194. [PubMed: 16084730]

22. Buratti E, Baralle FE. Front Biosci. 2008; 13:867. [PubMed: 17981595]

23. Buratti E, et al. J Biol Chem. 2005; 280

24. Mishra M, et al. Acta Neuropathol. 2007; 114:81. [PubMed: 17569064]

25. Abhyankar MM, Urekar C, Reddi PP. J Biol Chem. 2007; 282

26. Rollinson S, et al. Neurosci Lett. 2007; 419:1. [PubMed: 17434264]

27. Schumacher A, et al. Neurobiol Aging. 2007

28. Gijselinck I, et al. Neurobiol Aging. 2007

29. Materials and methods are available as supporting material on Science Online

30. This publication is dedicated to the memory of Dr Steven Ackerley, an outstanding young scientist. We would like to thank the patients and families who have contributed to this project and to Barbara Coote, Caroline Cecere, Joyce Gardham, the MRC Neurodegenerative Diseases Brain Bank and the Australian MND DNA Bank for assistance in sample collection. This work was supported by grants from: The Wellcome Trust, EU contract LSHM-CT-2003-503330 (APOPIS), American ALS Association, Motor Neurone Disease Association UK, Medical Research Council UK, the Middlemass family, Jack Cigman, King's College Hospital Charity, The Psychiatry 
Research Trust of the Institute of Psychiatry and the Motor Neuron Disease Research Institute of Australia (MNDRIA). 


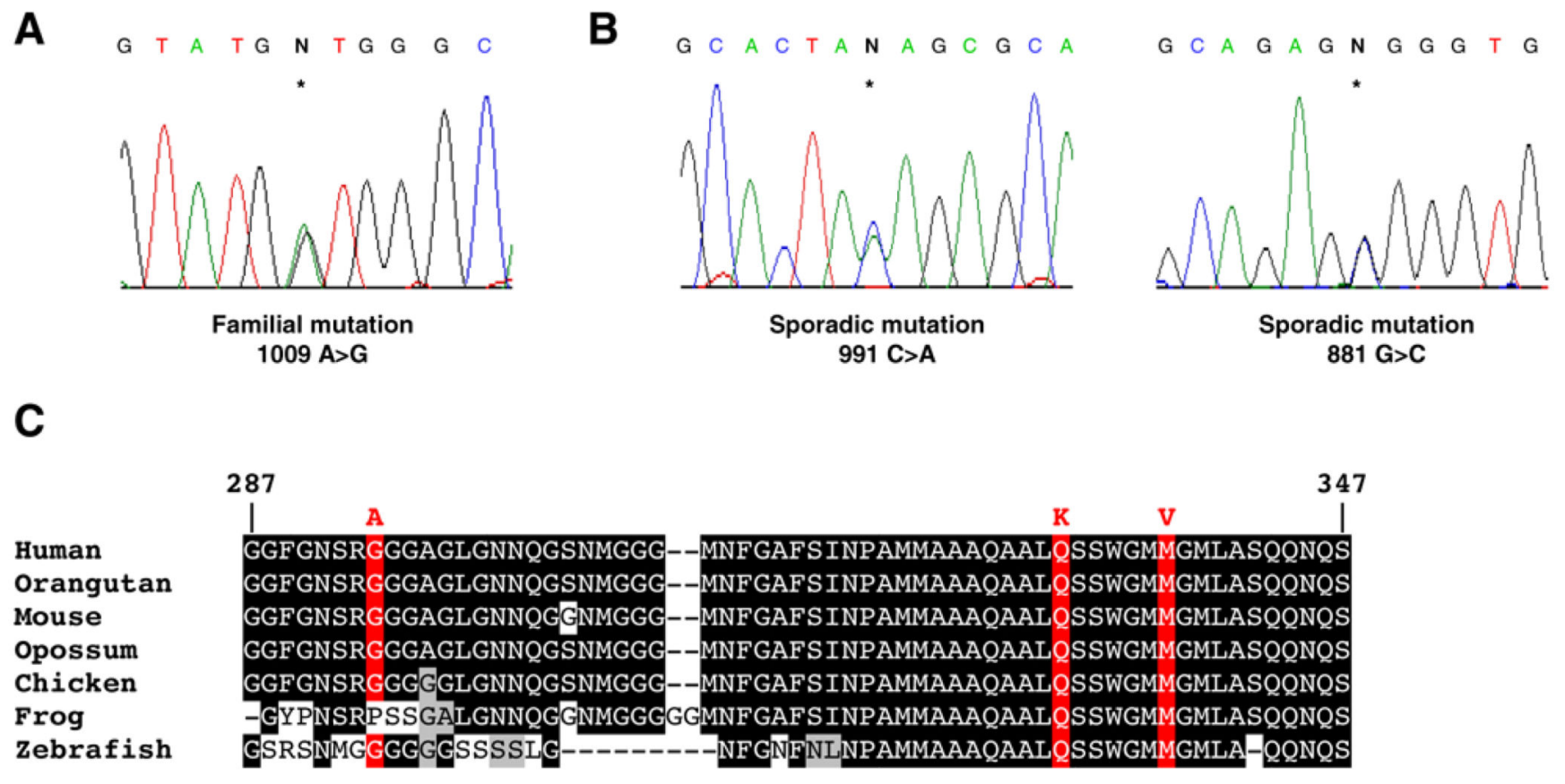

Fig. 1.

Identification of three missense mutations in exon 6 of TARDBP. (A) DNA sequence demonstrating a single base substitution (asterisk) changing the wild-type adenine at 1009 to guanine, substituting valine for methionine (M337V). (B) Two additional mutations were found in sporadic ALS cases; $991 \mathrm{C}>\mathrm{A}$ substituting lysine for glutamine (Q33 1K), and 881 $\mathrm{G}>\mathrm{C}$ substituting alanine for glycine (G294A). (C) A sequence alignment of amino acids 287-347 of TDP43 from diverse vertebrate species is shown. Identical amino acids have a black background, similar amino acids are grey and mutation sites are red. 


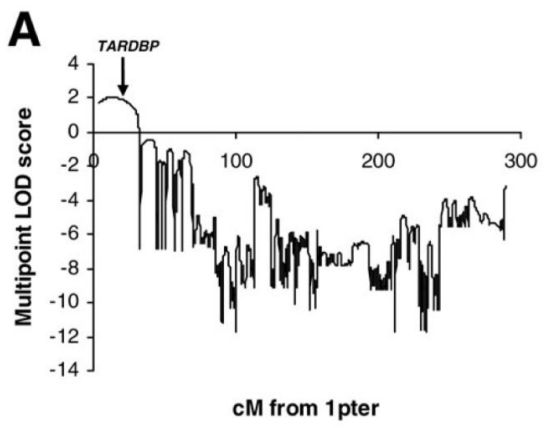

C

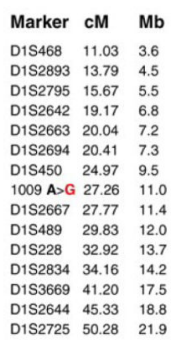

$\begin{array}{lll}\text { D1S2644 } & 45.33 & 18.8 \\ \text { D1S2725 } & 5028 & 21.9\end{array}$

$\begin{array}{lll}D 1 S 468 & 11.03 \quad 3.6\end{array}$ \begin{tabular}{lll} 
D1S2893 & 13.79 & 4.5 \\
\hline
\end{tabular} D1S2795 $15.67 \quad 5.5$ $\begin{array}{lll}D 1 S 2642 & 19.17 \quad 6.8\end{array}$ $\begin{array}{lll}D 1 S 2663 & 20.04 & 7.2\end{array}$ \begin{tabular}{lll}
$D 1 S 2694$ & 20.41 & 7.3 \\
\hline
\end{tabular} D1S450 $24.97 \quad 9.5$ D152567 $27.77 \quad 11.4$ $\begin{array}{lll}D 15489 & 29.83 & 12.0\end{array}$ D15228 $3292 \quad 137$ $\begin{array}{lll}D 1 S 2834 & 34.16 & 142\end{array}$ $\begin{array}{lll}\text { D1S3669 } & 41.20 & 17.5\end{array}$ D1S2644 $45.33 \quad 18.8$ $\begin{array}{lll}D 1 S 2725 & 50.28 & 21.9\end{array}$

$\begin{array}{lll}D 1 S 468 & 11.03 \quad 3.6\end{array}$ $\begin{array}{lll}D 1 S 2893 & 13.79 & 4.5 \\ \text { D1S2795 } & 15.67 & 5.5\end{array}$ $\begin{array}{lll}D 1 S 2642 & 19.17 & 6.8\end{array}$ $\begin{array}{lll}D 152663 & 20.04 \quad 72\end{array}$ $\begin{array}{lll}D 1 S 2694 & 20.41 & 7.3\end{array}$ $\begin{array}{lll}D 1 S 450 & 24.97 & 9.5\end{array}$ 1009 A $>$ G $27.26 \quad 11.0$ $\begin{array}{lll}D 1 S 2667 & 27.77 & 11.4\end{array}$ \begin{tabular}{lll}
$D 1 S 489$ & 29.83 & 12.0 \\
\hline & &
\end{tabular} $\begin{array}{lll}D 1 S 228 & 32.92 & 13.7\end{array}$ $\begin{array}{llll}D & 152834 & 34.16 & 13.2\end{array}$ $\begin{array}{llll}D 152644 & 45.33 & 18.8\end{array}$ $\begin{array}{llll}0152725 & 50.28 & 21.8\end{array}$

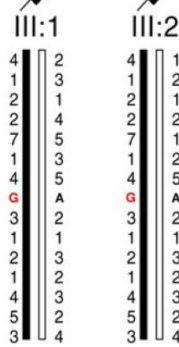

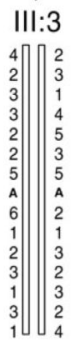

B

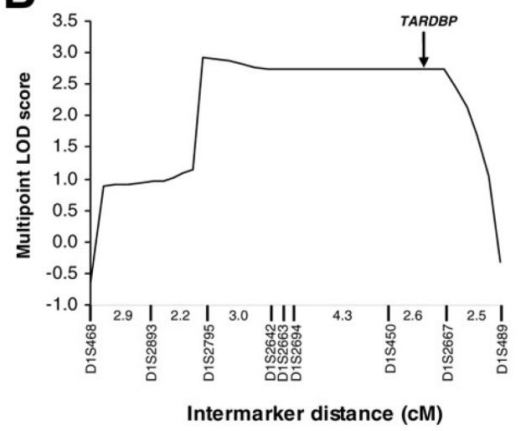

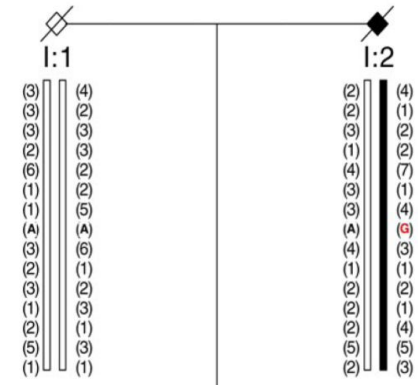
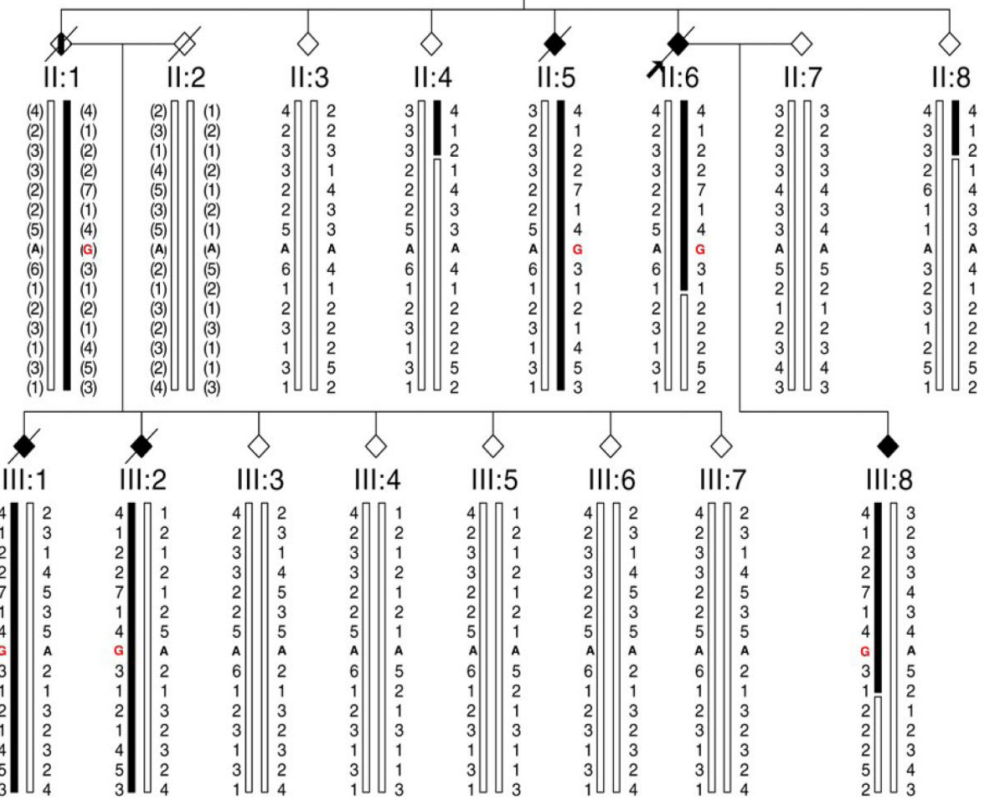

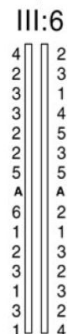

III:7

III:8

Fig. 2.

Mutation in TARDBP cosegregates with disease in kindred ALS85 (A) Linkage analysis of Affymetrix 10K SNP array data demonstrated linkage to chromosome 1p36. (B) Linkage to the region containing TARDBP was confirmed using microsatellite markers. (C) The ALS85 pedigree is shown. Affected individuals are indicated by black symbols. The proband is indicated by the arrow. Unaffected individuals have open symbols. II. 1 (vertical bar) was an obligate carrier with an anecdotal history of ALS. Slashed symbols indicate deceased subjects. Gender has been omitted for confidentiality. Microsatellite markers are shown in 
chromosomal order with their genetic and physical locations. Genotypes for each individual are given with inferred genotypes in parentheses. The haplotype segregating with disease is indicated by the black bar. The $1009 \mathrm{~A}>\mathrm{G} T A R D B P$ mutation is indicated by a red "G". 
A
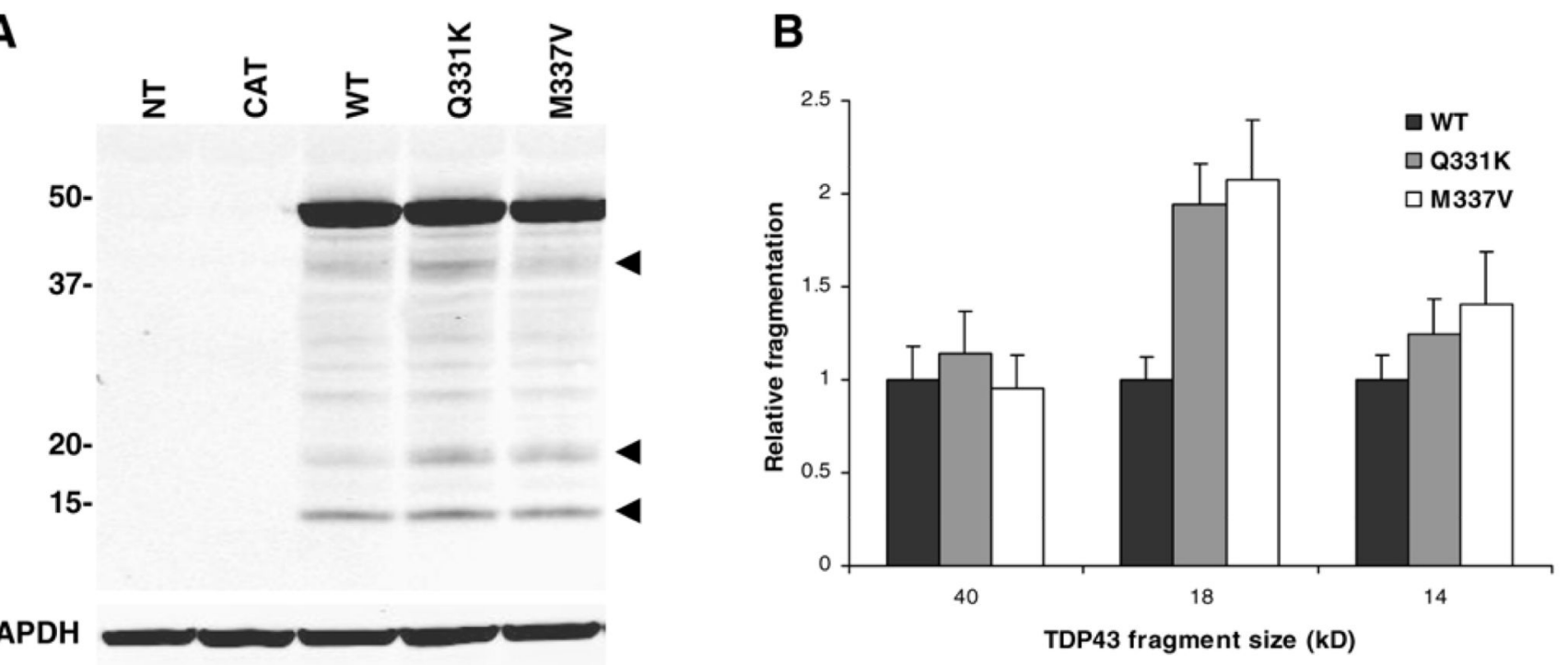

Fig. 3.

Immunoblots of cytoplasmic fractions from $\mathrm{CHO}$ cells transiently transfected with wild-type and mutant TDP-43. (A) Probing for the N-terminal myc tag demonstrating numerous fragments which were absent in controls (NT, non-transfected, and CAT, chloramphenicol acetyl transferase transfected). The dominant bands were at 40, 18 and $14 \mathrm{kD}$. (B) Quantitative analysis of myc-immunoreactive bands. Densitometry of bands was conducted using Odyssey. Results are presented as mean and standard error of the mean of eight experiments. 

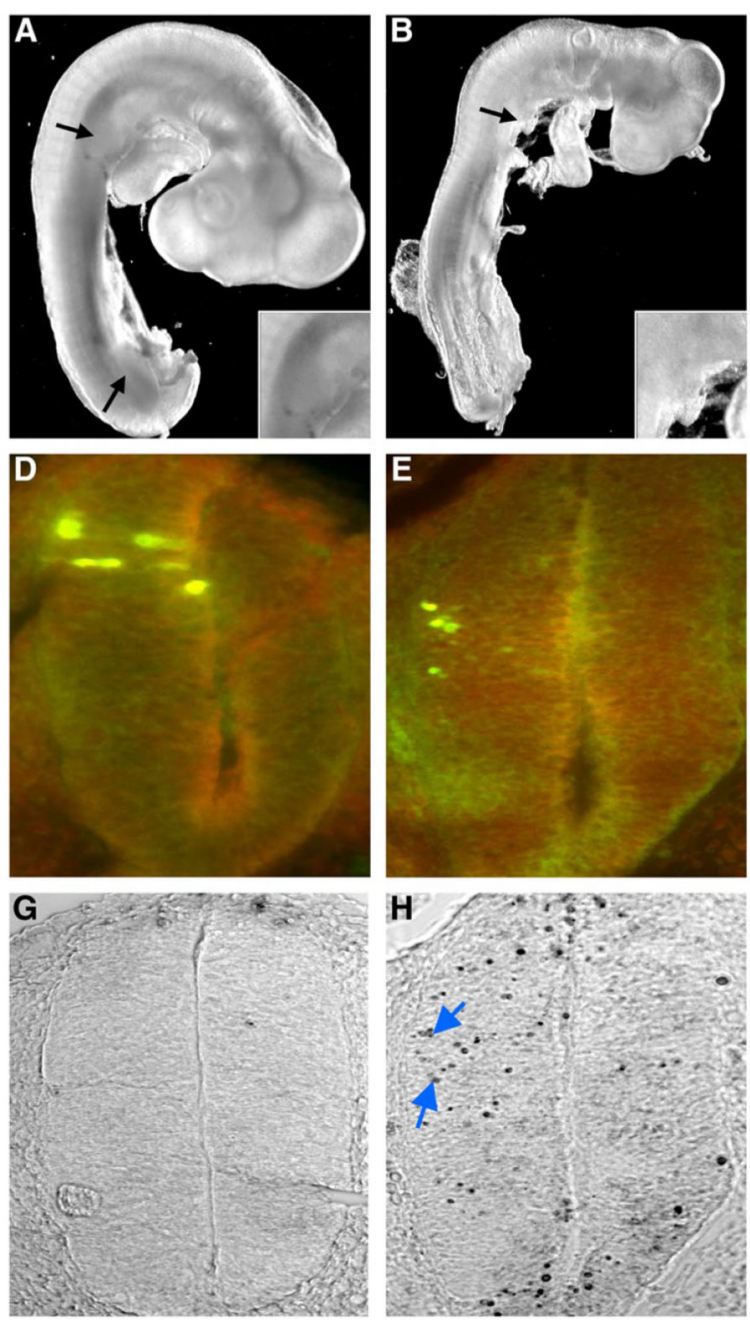
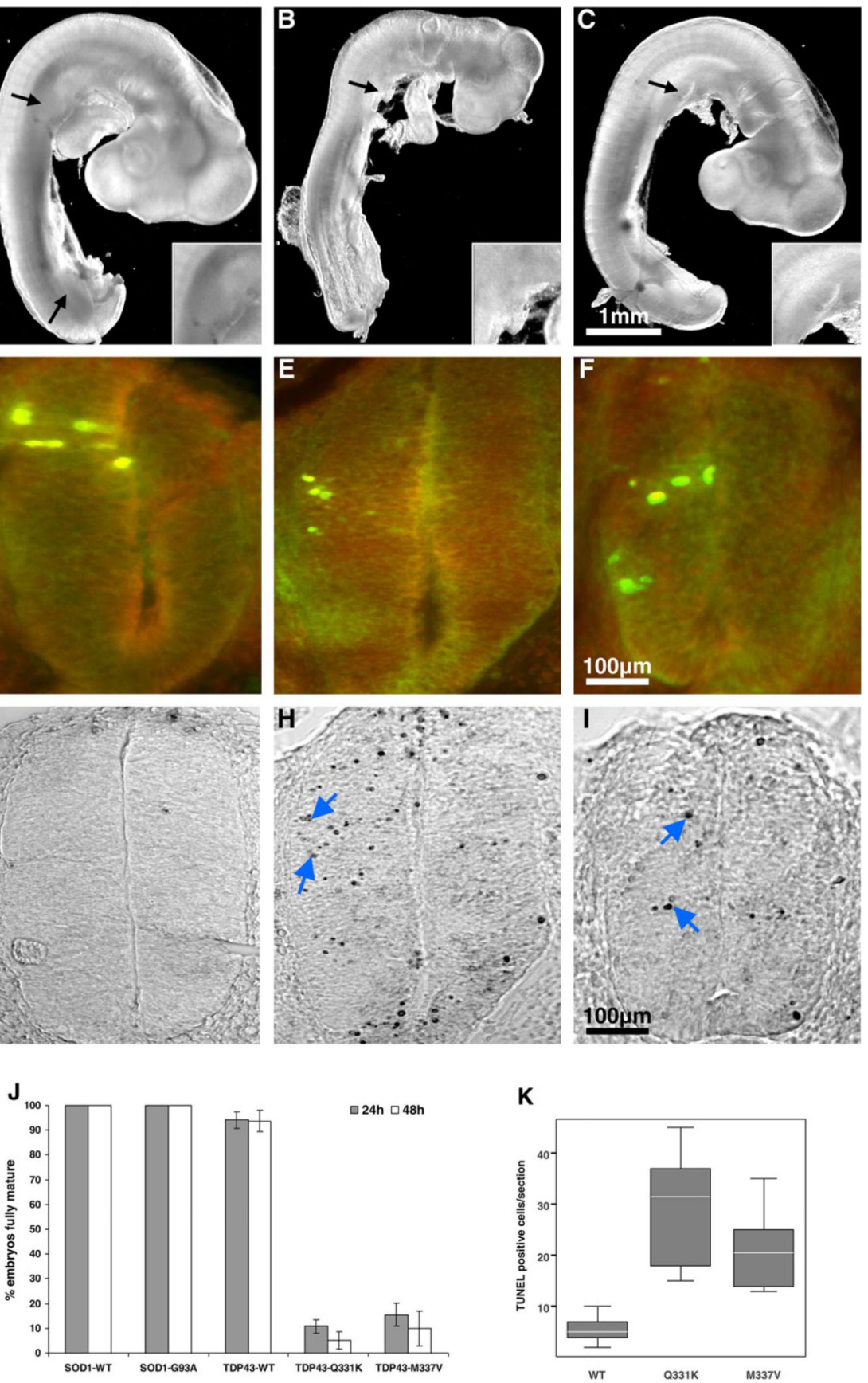

Fig. 4.

Mutant TDP-43 causes chick embryonic developmental delay. Chick embryo spinal cords were transfected with plasmids encoding wild-type TDP-43 (A, D, G), mutant Q331K (B, E, H) or M337V (C, F, I). Embryo images and sections shown were taken 24 hours post electroporation. Embryos expressing TDP43 WT developed normal limb (black arrows) and tail buds (A) while those expressing mutant TDP-43 did not (B, C). Magnified images of upper limb buds are shown in insets. (J) The percentage of embryos that matured normally (reached HH stage 15 or 17 after 24 hours and 48 hours respectively) is shown. Data were 
generated from analysis of 49 embryos in each treatment group. (D-F) Transverse sections of chick spinal cord double-stained with anti-HA (green) and anti-myc (red) antibodies to the tags on transfected TDP-43 constructs. Unilateral TDP-43 construct expression in spinal cord neural cells occurs following embryo electroporation. The majority of the cells in the transfected region have the characteristic phenotype of neuroepithelial cells. (G-I) TUNEL staining of sections shown in D-F demonstrated apoptotic nuclei (blue arrows). (K) Quantification of apoptosis. TUNEL positive cells in five sections per embryo (two embryos per transfection) were counted. 